\title{
Desarrollo de la cultura de la paz y la convivencia en el ámbito municipal: La mediación comunitaria
}

\author{
Ramón AlZATE SÁEZ DE HEREDIA \\ Universidad del País Vasco/Euskal Herriko Unibertsitatea \\ ramón.alzate@ehu.es \\ Itziar FERNÁNDEZ VILLANUEVA \\ Universidad Complutense de Madrid \\ icfernan@ucm.es \\ Cristina MERINO ORTIZ \\ Universidad del País Vasco/Euskal Herriko Unibertsitatea \\ cristina.merino@ehu.es
}

Recibido: 04/06/2012

Aceptado: 13/11/2012

\begin{abstract}
Resumen
Conceptualmente, una cultura de paz debería entenderse no como una utopía libre de conflicto, sino como una cultura en la cual los individuos, los grupos y las naciones tienen relaciones cooperativas y productivas unas con otras, y en dónde los conflictos, que surgen inevitablemente, se manejan de forma constructiva. Proponemos que los centros de mediación comunitaria deben adaptarse a la complejidad de los conflictos del mundo actual y responder a ellos con variados enfoques y metodologías: asesoramiento en resolución de conflictos, mediación, técnicas de intervención con grandes grupos, etc.
\end{abstract}

Palabras clave: cultura de paz, mediación comunitaria, asesoramiento, grandes grupos

Development of culture of peace and coexistence in the municipal level: CommunityMediation

\begin{abstract}
Conceptually, a culture of peace should be understood not as a conflict-free utopia, but as a culture in which individuals, groups and nations are cooperative and productiverelationships with each other, and where the conflicts that inevitably arise, are handledconstructively. We propose that community mediation centers must adapt to the complexity of conflicts in the world and respond to them with various approaches andmethodologies: advice on conflict resolution, mediation, intervention techniques with large groups, etc.
\end{abstract}

Keywords: culture of peace, community mediation, counseling, large groups. 


\section{Referencia normalizada}

Román Marugán, P. (2013). “La mediación política: concepto, procesos y problemáticas”. Política y Sociedad, Vol.50 núm. 1: páginas. 179-194

Sumario: 1.Cultura de paz. 2. Cultura de paz y Mediación Comunitaria. 3. Propuesta de un Centro Municipal de Transformación de Conflictos. 4. Asesoramiento en Resolución de Conflictos. 5.Intervención en grandes grupos. 6.Referencias bibliográficas

\section{Cultura de paz}

Como dijo Federico Mayor Zaragoza en las V Jornadas Internacionales de Cultura y Paz de Gernika: "La transición de una cultura de guerra a una cultura de paz quiere decir transición de las sociedades en las que el poder es garante de la seguridad a una sociedad que respalda pautas de comportamiento que tratan los desacuerdos y conflictos sin el recurso a la violencia. Esto significa una cultura de democracia que alienta y promueve la participación ciudadana, tanto en cuestiones nacionales, como internacionales; que reemplaza una autoridad jerárquica dominada por hombres por una igualdad entre hombres y mujeres en todos los niveles y define al poder como cooperación. Construye la "paz en las mentes de los hombres" y mujeres enlazando las personas en redes globales de intereses compartidos y comunidades locales con la internacional".

Hay quien dice que la causa en que radica la guerra es el conflicto, pero el conflicto es algo inherente a la vida humana, el resultado de que cada persona es algo único. En la ausencia del conflicto no habría enmienda alguna a la injusticia, convicción, ni creatividad. Sin embargo, si se permite que el conflicto degenere en violencia, se vuelve destructivo. El conflicto nunca puede resolverse definitivamente por medio de la violencia.

La clave de la cultura de paz es la transformación de la competición en cooperación, con lo que el conflicto se trata de manera que todos los involucrados se beneficien. Esto exige la incorporación de las partes en conflicto y de sus conflictos en el mismo proceso de desarrollo. Resumiendo, cabe decir que cultura de paz es el tratamiento del conflicto compartiendo el desarrollo.

De manera creciente se reconoce que podemos y debemos, transformar los valores, actitudes y comportamientos de la sociedad para pasar de la cultura de la violencia dominante a una nueva y evolucionada cultura de paz. Esta transformación requiere la adquisición de nuevos valores, actitudes y comportamientos; es una tarea que implica profesores, los creadores de opinión, los líderes políticos, etc.

Aunque sus principios fundamentales son claros, la cultura de paz es un concepto complejo que está desarrollándose y creciendo en nuestros días, como resultado de la práctica. La cultura de guerra ha impregnado cada uno de los aspectos de nuestras vidas, incluso en modos que no podemos ni imaginar. Del mismo modo, una cultura de paz transformará todos los rasgos del comportamiento humano, tanto 
a nivel individual como institucional, en formas que hoy en día son imprevisibles (Adams, 1995). El movimiento de una cultura por la paz, como un gran río, está alimentado por distintos riachuelos -diferentes tradiciones, culturas, lenguajes, religiones y perspectivas políticas-, su objetivo es un mundo en el que dicha diversidad de culturas vivan unas junto a otras en una atmósfera marcada por el entendimiento intercultural, la tolerancia, y la solidaridad.

Algunos podrían definir la cultura de paz como ausencia de conflicto, pero tal definición es demasiado pasiva e inconsistente con un mundo rico en diversidades. En un mundo diverso, el conflicto es, y será, parte de la vida misma. En vez de evitar y temer el conflicto, debemos aprender a apreciarlo y cultivar sus aspectos positivos y no violentos. Los conflictos son inevitables, necesarios, y pueden tener aspectos beneficioso; ayudan al desarrollo, a la identidad, a la reflexión y a la mejora, en general, del individuo y la sociedad. Pero los beneficios dependen de nuestra habilidad para enfrentarnos a los conflictos, para resolverlos de manera justa y para prevenir manifestaciones destructivas y violentas.

\section{Cultura de paz y Mediación Comunitaria}

Uno de los ámbitos fundamentales en el trabajo por la construcción de una cultura de paz, es el local, aquello que llamamos el ámbito comunitario, constituido por un diverso grupo de individuos que comparten una localización geográfica y que pueden compartir preocupaciones sociales, políticas y económicas comunes.

Las palabras de Elise Boulding (1986) reflejan e inspiran este papel comunitario de la construcción de paz: "Las culturas no se crean en los vestíbulos de los parlamentos y los palacios presidenciales; se crean localmente y sólo después se proyectan nacionalmente. Por ello, las culturas de la mediación y el hacer la paz deben comenzar localmente".

El papel de la resolución alternativa de conflictos y de la mediación comunitaria en particular es, precisamente, abordar desde la cercanía a las personas que están en conflictos la problemática de su tratamiento. Los sistemas tradicionales de resolver conflictos de los que nos hemos dotado en la cultura que domina nuestra sociedad, se han olvidado de la problemática real que envuelve al conflicto. Tratan, en el mejor de los casos, de abordar el conflicto basándose en los derechos y en los intereses de las partes, olvidándose de que la mayoría de los conflictos se producen en situaciones de relación que se extienden en el tiempo, por lo que la emociones y la calidad de la relación son tan, si no más, importantes.

Merry (1982), en este sentido, nos ofrece una descripción sucinta de la agenda de la mediación basada en la comunidad:

La mediación comunitaria nos ofrece medios para contrarrestar los estados de anomia, aislamiento, alienación y el miedo de la comunidad, facilitando la comunicación entre la gente que de otra manera permanecerían como conocidos distantes, incluso entrampados en relaciones de hostilidad y conflicto. 
La mediación puede ser un camino valioso de contrarrestar este aislamiento social ofreciendo mecanismos para que la gente se comunique unos con otros, rompiendo las hostilidades que están basadas en malentendidos. Incluso aunque hoy en día, el número de disputas resueltas sea pequeño, el impacto en la calidad de vida de la comunidad puede ser grande”

Siguiendo el modelo anidado del conflicto (Dugan, 1996) y su aplicación para la construcción de paz (Lederach, 1998), la mediación comunitaria tendría objetivos relacionados con los tres tipos fundamentales de conflictos, por lo que podría ser entendido como un enfoque fundamentalmente transformativo en sus cuatro niveles (personal, relacional, estructural, y cultural) y de construcción de paz. Según Dugan hay cuatro tipos de conflictos diferentes pero interrelacionados como se muestra en la figura adjunta. El primer tipo de conflicto, representado por el círculo interior son los conflictos relacionados con problemas concretos. Estos conflictos son los más fáciles de analizar (aunque no por ello los más fáciles de ser resueltos) y pueden ocurrir entre individuos o grupos de cualquier tamaño (disputas comerciales, problemas relacionados con ruidos, disputas entre arrendadores e inquilinos por la subida de los alquileres, etc.). En ocasiones, sin embargo, estos problemas no son la causa del conflicto. Los conflictos de relación son aquellos que surgen con cuestiones que tienen que ver con los patrones de interacción entre las partes y los sentimientos que tienen unos sobre otros (disputas reiteradas entre miembros de la comunidad de vecinos, conflictos entre distintas organizaciones ciudadanas, etc.).

En ocasiones, la causa del conflicto está más allá de las relaciones de las partes concretas que están en disputa, y están institucionalizadas dentro de la estructura del sistema social. Dugan hace una distinción dentro de este nivel entre conflictos relacionados en el sistema social en su más amplio sentido, y conflictos relacionados con las estructuras subsistémicas en donde ese sistema se refleja. Para algunos, este es un elemento fundamental para la mediación comunitaria. Es importante la identificación de esas capas del conflicto estructural subsistémico, que se refiere a esos conflictos que tienen su origen en las reglas, procedimientos y tradiciones de una organización social particular (municipio, escuela, organización,...) que son percibidas como injustas, antiguas, poco efectivas. 


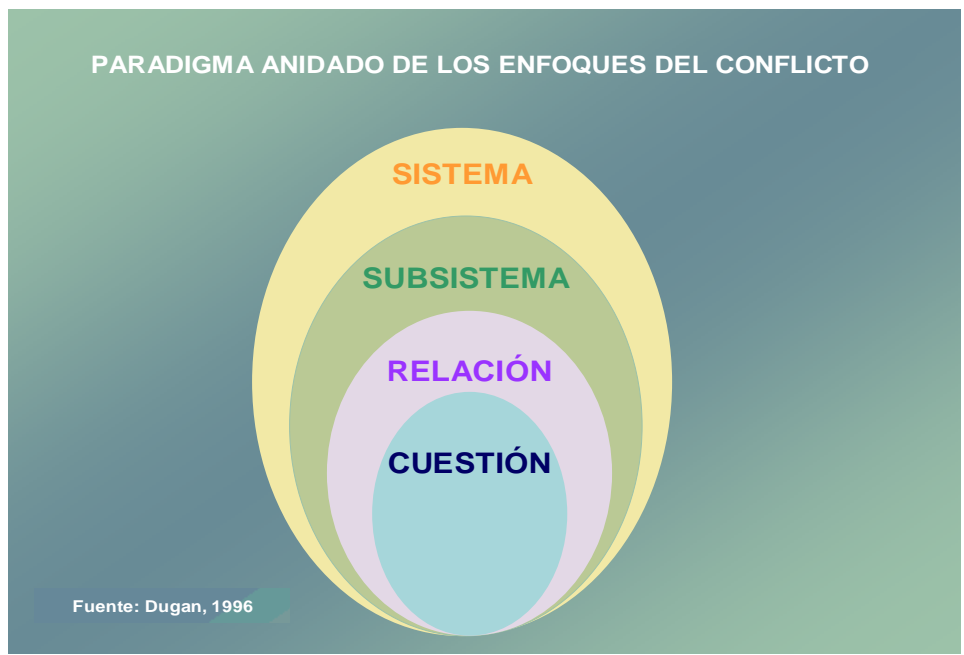

De acuerdo con este esquema, los centros de mediación comunitaria tienen objetivos relacionados con los tres primeros niveles (problemas, relaciones, subsistemacomunidad), afectando por extensión al cuarto nivel de la sociedad en su conjunto y su transformación cultural. La construcción de paz desde la mediación comunitaria se elabora desde la intervención en los conflictos puntuales (remitidos a los centros desde variadas vías), formación de la ciudadanía (voluntarios, organizaciones, políticos, etc...), e intervención en la transformación de los procesos de afrontamiento de problemas y decisiones, a través de procedimientos múltiples. Por definición, los centros de mediación comunitaria ofrecen servicios de mediación. Pero, muy pocos centros, si hay alguno, ofrecen sólo servicios de mediación. Además se ofrecen, por lo general, formación en resolución de conflictos y habilidades de comunicación, facilitación de reuniones, construcción de consensos, mediación en políticas públicas (relacionadas con la el medio ambiente y el urbanismo, por ejemplo), diseño de sistemas de resolución de conflictos para distintas organizaciones, conciliación (entendida como ayuda a llegar a soluciones a través de la comunicación entre varias partes, pero sin que las partes se encuentran juntas en ningún momento, en ocasiones se realiza telefónicamente), procesos de justicia restaurativa (círculos, conferencing...), y en algunos casos (aunque cada vez se tienda más a separarlo) arbitraje.

Es clarificador comentar que muchos de los programas de mediación comunitaria, de acuerdo con estas ideas, han asumido lo que denominamos el modelo transformativo de mediación, basado en el marco transformativo bosquejado por Bush y Folger en su libro The promise of Mediation (1994, 2005). Este modelo enfatiza dos importantes cambios que pueden ocurrir en mediación: el empoderamiento o fortalecimiento y el reconocimiento. El fortalecimiento se refiere a cualquier proceso donde los individuos o grupos ganan mayor control sobre materias que les afectan. A nivel psicológico el fortalecimiento se refleja en el incremento de la propia autoestima, confianza y percepción de autoeficacia. A nivel social, el forta- 
lecimiento implica ganar poder a través de la educación, politización y la acción colectiva. Usamos el término reconocimiento para recordar a las partes en conflicto que eviten estar centrados en sí mismas, y por el contrario reconocer la situación de la otra parte: el reconocimiento ocurre cuando las parte eligen voluntariamente mostrarse más abiertas, atentas, empáticas, y sensibles a la situación de la otra parte. Es obvia, la repercusión que la utilización de procedimientos de intervención en conflictos basados en esta perspectiva transformativa, tiene sobre la calidad de la convivencia ciudadana.

\section{Propuesta de un Centro Municipal de Transformación de Conflictos}

El planteamiento central de este apartado es proponer la necesidad de que los centros tradicionales de mediación comunitaria amplíen el ámbito de su intervención más allá de sus servicios de mediación comunitaria o social. Proponemos la creación de Centros Municipales (o Ciudadanos) de Transformación de Conflictos (CMTC) que sean agentes dinamizadores de la difusión de la Cultura de Paz, en el entorno comunitario en donde tienen su sede, desarrollando su actividad a través de un amplio espectro de proyectos que vayan desde la prevención a la intervención en crisis, desde el asesoramiento en resolución de conflictos (siendo parte aliada de las personas que necesitan abordar positivamente sus conflictos) a las intervenciones en grandes grupos (con el objetivo de lograr el compromiso y participación de la ciudadanía en los asuntos públicos que afectan su vida), pasando por las funciones tradicionales de formación y mediación comunitaria (Alzate, 2008).

En el esquema que desarrollamos a continuación, observamos de manera gráfica cuáles serían las funciones de dicho CMTC (de manera similar a cómo está planificado en el Peninsula Conflict Resolution Center del condado de San Mateo, California).

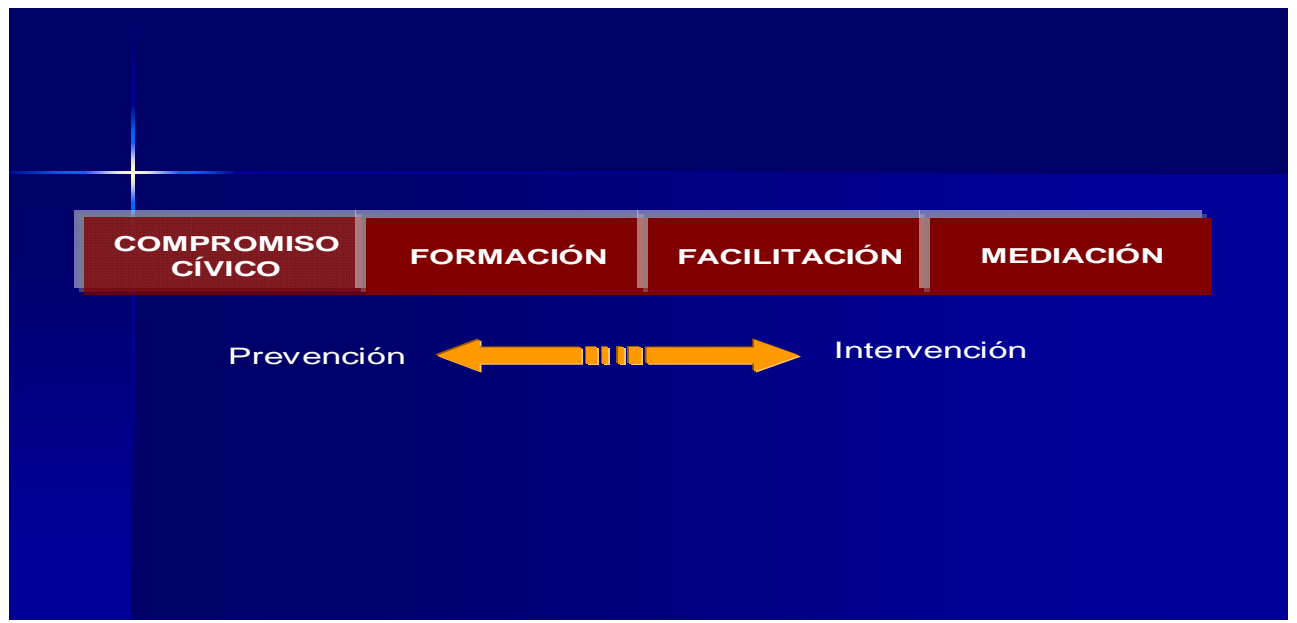




\section{COMPROMISO CÍVICO}

- Iniciativas diseñadas para incrementar la implicación activa de los ciudadanos en asuntos que afectan a sus vidas

- Incluye:

- Voluntariado

- Votación

- Organización Comunitaria

- Asesoramiento

- Diálogo

- Promoción del uso de la Resolución de Conflictos

- Estrategia principal es el uso de la promoción del diálogo público

\section{FORMACIÓN}

- Difusión de la Resolución de Conflictos

- Programas para padres y madres

- Entrenamiento avanzado para mediadores

- Programas para actores comunitarios (policía, voluntarios.....)

- Formación en Comunicación y Técnicas de Resolución de Conflictos

\section{FACILITACIÓN}

- Planificación de Estrategias

- Diseño de Procesos

- Facilitación

- Intervención en grandes grupos

- Toma de decisiones

- Diseño del futuro

- Resolución de conflictos

\section{MEDIACIÓN}

- Mediación Comunitaria

- Mediaciones Complejas Multiparte

- Mediación Familiar

- Intergeneracional

- Tercera Edad

A continuación nos detenemos brevemente en los aspectos menos tradicionales dentro de un Centro Municipal de Transformación de Conflictos: el asesoramiento en resolución de conflictos y las intervenciones con grandes grupos. 


\section{Asesoramiento en Resolución de Conflictos}

El asesoramiento en resolución de conflictos es un concepto que combina principios de la resolución de conflictos y principios del coaching. Es un proceso uno a uno confidencial y voluntario en el que los asesores trabajan con las personas para ayudarles a resolver disputas existentes y prevenir la ocurrencia de conflictos innecesarios. Puede aplicarse a un problema específico en el que el individuo está o ha estado implicado, o que anticipa lo que vaya a ocurrir. Pero también se usa para personas que quieren adquirir nuevas habilidades en general, o reforzar algunas ya existentes, pero no es necesario que se refieran a ningún conflicto específico. El asesoramiento en resolución de conflictos es, en su esencia, un método individualizado para ayudar a la gente a tratar los conflictos de manera más eficaz.

El asesoramiento es una técnica emergente en la disciplina de resolución de conflictos que ofrece una estrategia al profesional para aquellas situaciones en las que sólo está presente una de las partes del conflicto.

Los principios de la confidencialidad y autodeterminación, son semejantes en el ejercicio de cualquier procedimiento que se encuentre dentro del ámbito de la Resolución Alternativa de Disputas (RAD) y, por lo tanto, también del asesoramiento. Asimismo, hay muchas técnicas similares pero, también, algunas diferencias con la perspectiva más cerrada de la resolución de conflictos. Hay que señalar que los objetivos de las personas que participan en el asesoramiento, por lo general, suelen ir más allá de la resolución de un conflicto particular. En muchas ocasiones, las personas que buscan asesoramiento profesional pueden querer prevenir que una disputa escale de manera innecesaria, mejorar sus competencias en el manejo del conflicto, desarrollar mejores habilidades de comunicación, etc. Se trata, con frecuencia, más de manejar un conflicto que de resolverlo.

Hay ciertos riegos al acecho de todo profesional en Resolución de Conflictos, pero estos riesgos son especialmente reales en la práctica del asesoramiento, debido a su propia naturaleza (Nobel, 2005). En primer lugar el entrenador/asesor, no es un terapeuta. En el proceso de entrenamiento/asesoramiento, como en el resto de procesos de Resolución de Conflictos, es inevitable que las personas ventilen su versión de los hechos, sus preocupaciones y emociones. Por supuesto, el entrenador/asesor debe permitirlo (y como veremos en uno de los modelos presentados, recomendarlo) permaneciendo en una actitud de apoyo, aunque sin evaluarlo. A diferencia de la terapia o el counseling, el entrenador/asesor no explora experiencias pasadas o la génesis de los comportamientos conflictivos y respuestas emocionales. Esto no significa que no haga referencia a acontecimientos históricos que han tenido un impacto en la situación actual, sino que esta incursión en el pasado tiene sus limitaciones. El proceso está centrado en el futuro y, en consecuencia, el entrenador/asesor no actúa cuando lo que la persona necesita es trabajar asuntos emocionales no resueltos.

Tampoco es un mentor que da consejos. Un mentor, normalmente, es una persona con cierto ascendente que da información, consejos, o cualquier otro tipo de asistencia, basándose en su experiencia y capacidades. El entrenamien- 
to/asesoramiento, por el contrario, está basado en la autodeterminación, es decir, dirigido a que la persona sea la propia experta.

Por supuesto, el entrenador/asesor no es un mediador, ya que sólo hay una persona y se sitúa al lado de la parte, no en medio de las partes. Esto no impide que cuando una parte acude solicitando un proceso de mediación y la mediación no es posible, por negativa de la otra parte en conflicto, el mediador no pueda ejercer de entrenador/asesor.

Finalmente, tampoco hay que confundir el entrenador/asesor, con una persona que ejerce funciones de representante o agente (como por ejemplo en el derecho colaborativo). El entrenador/asesor no actúa como representante, no acude a hablar con la otra persona en nombre de la parte con la que trabaja, no participa en un proceso abogando por los intereses de la parte, no actúa como agente o representante en un proceso basado en los derechos. En definitiva, no habla por la parte implicada en ninguna circunstancia.

El asesor en resolución de conflictos ayuda a las personas a romper el ciclo negativo del conflicto (Community Boards y Alzate, 2000), caracterizado por la evitación y/o confrontación, que tan pernicioso resulta para las relaciones. Como hemos dicho, ésta ayuda puede ser improvisada y espontánea, en cualquier contexto en donde surge la conversación, o puede ser planificada (profesionalizada) cuando una persona, o varias, te invita a discutir una situación de conflicto y a que le asesores a través del proceso de resolución. Este asesoramiento, puede ser tan corto como 20 minutos, como durar varias reuniones, días, meses o incluso un año.

\section{Intervención en grandes grupos}

Los tiempos de las sociedades, comunidades y organizaciones estables y predecibles, han pasado, o están pasando a un ritmo vertiginoso. Vivimos en una sociedad en la que cada vez somos más conscientes de la diversidad de valores, procedencias étnicas y religiosas que existen en nuestro entorno. Esta nueva situación requiere, cada vez con más urgencia, que las comunidades y organizaciones evolucionen y se transformen en organismos cada vez más flexibles y con mayor capacidad de respuesta ante los numerosos cambios y retos que van surgiendo continuamente. Se requieren comunidades capacitadas para recoger información de su ciudadanía y que puedan planificar su futuro con rapidez, no al ritmo lento de la política tradicional. Esto no ocurría en el pasado, es un fenómeno nuevo al que hay que dar una respuesta.

Habitualmente en este mundo moderno, así como en el ámbito de la resolución de conflictos, buscamos desesperadamente respuestas y soluciones a las distintas situaciones y conflictos que se nos presentan en la vida cotidiana, en muchas ocasiones a través de los centros de mediación comunitaria. No queremos perder tiempo, queremos salir adelante de las situaciones difíciles lo antes posible, y en cuanto tenemos una respuesta, nos gusta pasar la información a los demás. Transmitimos las soluciones y los procesos que llevan a ellas a través de las publicaciones, semi- 
narios, cursos, conferencias, etc. Este enfoque, no hay duda de que es eficaz y conveniente en determinadas circunstancias, y para determinados problemas, pero para los problemas complejos que caracterizan los desafíos que la humanidad enfrenta en el siglo XXI, no parecen apropiados por diversas razones (Bojer, Knuth y Magner, 2006).

En primer lugar, vivimos en un mundo cada vez más complejo, donde las respuestas tienen una vida muy corta. Kahane (2004) señala que los problemas difíciles están caracterizados por tres tipos de complejidad. En primer lugar la complejidad dinámica que significa que la causa y el efecto están distantes en el espacio y en el tiempo. Para afrontar este tipo de complejidad se debe adoptar un enfoque sistémico sobre el problema y la solución. La complejidad social se refiere a la existencia de muchas asunciones y puntos de vista diferentes sobre el problema o conflicto, y que, por lo tanto, no hay ninguna entidad que posea exclusivamente la visión del problema. Esta complejidad requiere un enfoque participativo. Finalmente, se da una complejidad generativa, que significa que las soluciones del pasado no funcionan más, y que el problema es impredecible y en continuo cambio, lo que requiere un enfoque creativo.

En segundo lugar, hay una clara constatación generalizada de que las personas quieren resolver sus propios problemas. Cuando se importa o impone una respuesta a sus problemas desde el exterior, se produce una resistencia que hace, con frecuencia, que la respuesta o solución fracase. Esta circunstancia puede deberse, parcialmente, a que la respuesta no es de hecho adecuada al contexto particular, pero también, y en ocasiones principalmente, porque las personas que no han participado en la solución o a las que no se ha consultado, no sienten las soluciones como propias. Los seres humanos tienen una tendencia interior profunda que los impulsa hacia la libertad y la autodeterminación y, dadas las circunstancias adecuadas, las personas tienen más recursos de los que nos imaginamos a la hora de encontrar sus propias respuestas. El éxito a la hora de implementar intervenciones sobre conflictos sociales depende, en gran medida, del sentido de propiedad y motivación de los implicados y no de la brillantez de la idea.

Aunque sólo fuera por estas dos razones, deberíamos dedicarnos más a hacer preguntas y a hablar y escucharnos los unos a los otros.

Este tipo de problemática ha sido abordada tanto por el trabajo organizacional como por la resolución de conflictos, aunque no exista mucha tradición en el afrontamiento de estas nuevas realidades, y no hayan sido incorporadas a lo que a lo que consideramos su sitio natural, los centros de mediación comunitaria -a los que denominaríamos Centros Comunitarios de Transformación de Conflictos. El punto de inflexión fue el año 1992, con la publicación de un número especial del Journal of Applied Behavioral Science. Antes de esa fecha existían experiencias en las que unos pocos profesionales desarrollaban procedimientos innovadores (p.e., Owen con el espacio abierto y Cooperrider con los diálogos apreciativos), pero no existía la conciencia de que estaba surgiendo un nuevo ámbito en la resolución y manejo de los conflictos. A partir de ese momento comenzaron a desarrollarse nuevos 
procesos y métodos para trabajar con grandes grupos con el objetivo de producir cambios a nivel de todo el sistema.

En la última década se vienen utilizando estos métodos con distintos objetivos, pero, en general, con la intención de conseguir cambios a gran escala: cambio en la dirección estratégica de un negocio, transformación de los procesos de trabajo para conseguir mayor productividad, tomar decisiones o solucionar problemas/conflictos en una comunidad, o a nivel sistémico en cualquier otra instancia.

El elemento filosófico central de estos procedimientos es la inclusión o invitación a participar en el diálogo y/o en el proceso de toma de decisiones, a todas las partes afectadas por la decisión o por la acción a tomar. Si se trata de una organización empresarial, deben estar presentes la dirección, los empleados/as los clientes, los suministradores, etc.; si la cuestión se plantea en el ámbito escolar los participantes deberán ser, la administración, el profesorado, los/as estudiantes, los padres/madres, las fuerzas sociales...; si el reto se da en una comunidad, podrían estar, dependiendo de la cuestión, las asociaciones de vecinos, los partidos políticos, la policía, representantes de las distintas sensibilidades ciudadanas, etc. La cuestión central es que los que van a verse afectados por las decisiones, tienen derecho a estar representados en el proceso. El tamaño del grupo varía con los procedimientos (de 10 a más de 1.000), pero en general, el número vendrá determinado por la "masa crítica" de gente necesaria para lograr un cambio real (Bunker, 2006), aunque cuestiones presupuestarias o de limitación de espacios restringirían nuestras posibilidades.

Bunker y Alban (1992, 1997, 2006), proponen una clasificación de los distintos procedimientos y métodos en función de los resultados producidos:

1. Métodos para crear juntos el futuro.

2. Métodos para diseñar el trabajo.

3. Métodos para toma de decisiones y resolución de conflictos.

En el ámbito de la resolución de conflictos tratamos fundamentalmente de los métodos de la primera y la tercera categoría, ya que la segunda categoría está más relacionada con el aumento de la productividad en empresas y organizaciones, que, siendo importantes en el mundo actual de la economía globalizada, no son el objeto de nuestro trabajo.

\section{Métodos para crear juntos el futuro}

Todos estos métodos reúnen a todo el sistema que se trate, para definir el futuro y establecer objetivos a conseguir. Algunos de los más conocidos son:

1. The Search Conference.

2. Future Search.

3. AmericaSpeaks.

4. Appreciative Inquiry Summit. 


\section{Métodos para el diálogo y la toma de decisiones.}

1. Simu-Real.

2. Open Space.

3. The World Café.

4. Public Conversation Project.

Estos métodos se han ido desarrollando creando procesos que permiten manejar o resolver distintos tipos de conflictos a nivel comunitario y organizacional. Bunker (2005) enumera ocho principios que contribuyen a la efectividad de las intervenciones con grandes grupos:

1. Centrarse en lo compartido (common ground), en áreas de acuerdo más que en las diferencias o en los intereses competitivos.

2. Racionalizar el conflicto. Esto significa que hay que reconocer el conflicto y aclararlo, más que ignorarlo o negarlo. Aceptar que hay desacuerdos, y avanzar hacia áreas de acuerdo.

3. Expandir los puntos de vista egocéntricos sobre la situación que tienen las personas exponiéndolas a muchos puntos de vista en un grupo heterogéneo que trabaja colaborativamente en un espíritu de grupo.

4. Fomentar el desarrollo de relaciones personales a través de estructuras tales como pequeñas mesas en donde se reúnen grupos reducidos para intercambiar información y puntos de vista en actividades estructuradas. (Una percepción de compartir relaciones personales ayuda a manejar las diferencias).

5. Permitir tiempo para que se reconozca la historia del grupo con el conflicto, así como sus sentimientos, antes de pretender que el grupo comience a trabajar colaborativamente.

6. Manejar las expresiones públicas de las diferencias y del conflicto. Tratar a todos los puntos de vista con respeto. Permitir que los puntos de vista de las minorías sean escuchados, pero que no dominen la discusión. Reservar tiempo para la expresión de los puntos de vista de la gente que se encuentra "en el medio", al igual que lo hacemos con los extremos.

7. Manejar el conflicto evitando temas incendiarios o asuntos que no pueden ser abordados en el tiempo disponible.

8. Reducir la jerarquía tanto como sea posible. Poner la responsabilidad del trabajo conjunto y del manejo del conflicto en la comunidad u organización para que la gente sea responsable de sus propias actividades.

Damos por supuesto que todos los métodos de intervención en grandes grupos, comparten una clara voluntad de usar el diálogo para producir o crear un cambio. 
Sin embargo, es conveniente considerar una serie de principios que deberían caracterizar todos los procesos de diálogo. Los autores y profesionales han establecido diferentes listas de principios, pero consideramos que las propuestas por Pruitt y Thomas (2007), recogen la esencia fundamental a todas ellas.

Los procesos de diálogo deberían distinguirse por:

\section{Inclusividad.}

La inclusión junto a la sunción asociada de implicación/responsabilidad es, para la mayoría de los profesionales el principio fundamental de los procesos de diálogo. Todos los diálogos asumen que cualquiera de las partes implicadas en una situación problemática pueden participar o ser representadas en el proceso de diálogo. Se entiende que la participación colectiva aporta los elementos claves que permiten tener la capacidad para afrontar los problemas. En cuanto a la implicación/responsabilidad se considera que para que los cambios sean sostenibles, las personas que pertenecen al sistema deben poseer un cierto sentido de "propiedad" del problema, del proceso para afrontarlo, y de las soluciones adoptadas. Para que se desarrolle en los afectados este sentido de implicación, se debe participar, de alguna manera, en el proceso de cambio.

\section{Corresponsabilidad.}

Este principio ilustra que los proceso de diálogo no son un instrumento de un solo protagonista. Por ejemplo, un responsable municipal, que convoca a un grupo de ciudadanos para hablar con ellos y consultar algunos puntos y, tras esta consulta "superficial”, seguir con su propia dinámica. Un diálogo es un intercambio en el que las personas están implicadas en un nivel de igualdad, en el que la comunicación se mueve en doble dirección, y en el que básicamente no existen desequilibrios. Las personas implicadas tienen la posibilidad de influir sobre su propio futuro.

\section{Aprendizaje.}

Un profesional del ámbito de las conversaciones públicas comentó en cierta ocasión: "un diálogo no trata del acto físico de hablar, sino de mentes que se despliegan”. Esta característica llama la atención sobre la calidad de la interacción en los procesos de diálogo y los distingue de los "falsos" diálogos en donde la comunicación tiene una sola dirección y de los debates y negociaciones en donde los participantes se centran en ganar lo máximo posible para uno mismo. Algunos autores se refieren a esta característica como "apertura", en el sentido de que los participantes se abren para oír y reflexionar sobre lo que los otros tienen que decir y sobre lo que uno mismo está diciendo, para así lograr una nueva comprensión y perspectiva sobre el asunto tratado.

William Isaacs (1999) describió una serie de comportamientos y habilidades claves para crear este tipo de interacción: escuchar, sin prejuicios ni resistencias; respetar, conciencia de la integridad de la posición de la otra parte, y de la imposibilidad de comprenderla completamente; suspensión, suspender las propias certezas, asunciones y juicios.

Lo que se aprende en los ambientes creados por los distintos procedimientos de diálogo, procede en gran medida de la característica inclusiva del diálogo, que junta 
personas que no lo hubieran hecho en circunstancias normales y que incluso pueden estar en conflicto.

\section{Humanidad.}

"A través del diálogo nuestra inteligencia natural es capaz de revelarse. Nuestra humanidad tiene la posibilidad de reconocerse" (Von Meijenfeldt, Santino y Angeby, 1999). Junto a la disposición para aprender, esta característica de humanidad es la que más claramente distingue los procesos de diálogo con otro tipo de interacciones. Por humanidad se entiende el modo en el que nos comportamos con los otros cuando estamos en un diálogo verdadero. La humanidad requiere empatía, toma de perspectiva -habilidad para ponernos en la posición del otro-, y autenticidad aspecto que Isaacs (1999) le llevaba a establecer su cuarta habilidad clave para el diálogo, manifestar, decir la verdad desde uno mismo, lo que uno piensa y es realmente.

Crear un entorno que promueva el aprendizaje y este tipo de interacción humana, es un elemento central de todos los métodos de diálogo y deliberación. Algunos métodos se refieren a este aspecto como el de la creación de un "espacio seguro", poniendo un énfasis especial en la creación de un ambiente de confianza que lo posibilite.

\section{Perspectiva a largo plazo.}

En el tipo de problemas con los que trabajan los métodos de intervención con grandes grupos, un elemento fundamental es la sostenibilidad de las soluciones. Una de las características definitorias de los diálogos que tienen lugar en ellos es, precisamente, la perspectiva a largo plazo que posibilita alcanzar tal sostenibilidad. Aunque hay muchos problemas que requieren una rápida intervención -violencia, miseria, etc.-, los diferentes métodos de intervención de los que estamos hablando, se centran en los patrones de comportamiento y de relaciones que subyacen a las crisis particulares. Sólo si se trabaja a estos niveles podemos encontrar soluciones y cambios sostenibles en el tiempo. Para este tipo de problemas no hay soluciones rápidas,

Debemos, finalmente señalar que, en la actualidad, estos procedimientos comienzan a formar parte de los recursos utilizados habitualmente por muchos profesionales y deberían, sin ninguna duda, ser parte de los servicios que los centros de mediación comunitaria ofrecen. En los últimos años se han aplicado en una gran variedad de entornos -se usan en el mundo de la empresa, en los sistemas de salud, en la educación, en el gobierno, y a nivel comunitario. 


\section{Referencias bibliográficas}

Adams, D. (Ed) (1995): UNESCO and a Culture of Peace. Paris: UNESCO.

Alzate, R. (2008): "Mediación comunitaria, asesoramiento y procesos de participación ciudadana". En Jose Vasconcelos-Sousa (Ed), Campos da Mediaçao. Novos caminhos, novos desafios. Mediation fields. New Paths, new challenges, pp. 1932. Coimbra Ed. MinervaCoimbra.

Bojer, M.; Knuth, M. y Magner, C. (2006): Mapping dialogue: A research project profiling dialogue tools and processes for social change. South Africa: Partners for the Future Worldwide.

Boulding, E. (1986): "Presentación”. En J. Beer, Peacemaking in your neighbourhood: Reflections on an experiment in Community Mediation. Philadelphia: New Society Publishers.

Bunker, B.B. (2005): "Managing conflict through large-group methods". En M. Deutsch, P.Y. Coleman y E.C. Marcus (Eds), The handbook of conflict resolution. Theory and practice. San Francisco Jossey-Bass

Bunker, B.B. y Alban, B.T. (1992): Large group interventions. Journal of Applied Behavioral Science (special issue), 28 (4).

Bunker, B.B. y Alban, B.T. (2005): Large group interventions. Journal of Applied Behavioral Science (special issue), 41 (1).

Bunker, B.B. y Alban, B.T. (1997): Large group interventions. San Francisco: Jossey-Bass.

Bunker, B.B. y Alban, B.T. (2006): The handbook of large group methods. San Francisco: Jossey Bass.

Bush, R.A.B. y Folger, J.P. (1994, 2005): The Promise of Mediation. San Francisco: Jossey-Bass.

Community Boards y Alzate, R. (2000): Transformación del Conflicto: Curriculum para Bachillerato y Secundaria .Bilbao. Editorial Mensajero.

Dugan, M. (1996): A nested theory of conflict. A Leadership Journal: Somen in Leadership-Sharing the Vision, vol.1, 9-20.

Holman, P.; Devane, T. y Cady, S. (2007): The change handbook. The definitive resource on today's best methods for engaging whole systems. San Francisco: Berrett-Koehler.

Isaacs, W. (1999): Dialogue and the art of thinking together: A pioneering approach in communicating in business and in lifei. New York: Doubleday.

Kahane, A. (2004): Solving tough problems. San Francisco: Berrett-Koehler.

Lederach, J.P. (1998): Construyendo la paz: Reconciliación sostenible en sociedades divididas. Bilbao, Bakeaz/Gernika Gogoratuz.

Merry, S.E. (1982): The social organization of mediation in non-industrial societies: Implications for informal community justice in America. In The Politics of Informal Justice, 17-45. New York: Academic Press.

National Coalition for Dialogue \& Deliberation. www.thataway.org 
Noble, C. (2005): Conflict coaching -When it Works and when it doesn't. Presentación para Waves of Change, LEADR's $8^{\text {th }}$ International ADR Conference, Sydney, Australia.

Pruitt, B. y Thomas, P. (2007): Democratic dialogue: A handbook for practitioners. CIDA, IDEA, OAS, UNDP.

Von Meijenfeldt, R., Santiso, C. y Ängeby, M. (1999): Dialogue for democratic development: Policy options. Stockholm: International IDEA. 